\title{
1.
}

ARGONNE NATIONAL LABORATORY

P.O.Box 299

Lemont, Illinois

\section{THE "SMOOTH APPROXIMATION" TO HILL EQUATION \\ by}

L.C. Teng

Physics Division

January, 1956

Operated by The University of Chicago under

Contract $W-31-109-e n g-38$ 


\section{DISCLAIMER}

This report was prepared as an account of work sponsored by an agency of the United States Government. Neither the United States Government nor any agency Thereof, nor any of their employees, makes any warranty, express or implied, or assumes any legal liability or responsibility for the accuracy, completeness, or usefulness of any information, apparatus, product, or process disclosed, or represents that its use would not infringe privately owned rights. Reference herein to any specific commercial product, process, or service by trade name, trademark, manufacturer, or otherwise does not necessarily constitute or imply its endorsement, recommendation, or favoring by the United States Government or any agency thereof. The views and opinions of authors expressed herein do not necessarily state or reflect those of the United States Government or any agency thereof. 


\section{DISCLAIMER}

Portions of this document may be illegible in electronic image products. Images are produced from the best available original document. 
by

\section{C. Teng}

Symon" has developed a procedure for solving "approximately" the extended Hill equation.

$$
x^{\prime \prime}=f(x, \infty)
$$

where prime denotes differentiation with respect to $D$, and where $f(x, d)$ is periodic in $A$ with period $S$. This so-called "s mooth approximation" is analyzed, re-interpreted, and extended in this report.

Because of the periodicity of $f(x, A)$ in $A$, the solution of (1) will consist of a detailed periodic structure with period $S$, superimposed on a gross or "smoothed" structure. Our aim now is to find the differential equation whose solution $\bar{X}=\bar{X}(A)$ exhibits only the "s moothed" or gross structure of the solution $x=x(\mathrm{~A})$ of (1). Geometrically, the curve $\bar{X}=\bar{X}(\mathrm{~S})$ is simply that which passes "smoothly" through the points (reference points) $x(x S)(n=0, \pm 1$, $\pm 2, \pm 3, \cdots \cdots)$. The manner of smoothness in which this curve passes through the reference points is determined by the following criteria. Firstly, the "smoothed" curve obviously should coincide with the exact curve when the latter is itself truly smooth, $\dot{x}$.e., when $f(x, A)$ is independent of $A$. Secondly, if the detailed periodic structure of the "exact" curve is to be completely absent in the "smoothed" curve, the lattet should be independent of $S$, and its genetic differential equation should be independent of the specific choice of the reference point within a given period. Moreover, equation (1) being of the second order, determines a two parameter set of curves. The isomorphism between this set and the set of "smoothed" curves then requires the "s moothed" differential equation to be also of the second order. Thus to find the "smoothed" differential equation we need a three point local fitting.

If we denote the function $\mathcal{X}(S)$ at a typical reference point temporarily chosen as the local origin by $X_{0}$ and the slope of the "exact" curve at that point by $X_{0}^{\prime}$, then the values of $X$ at the neighboring reference points (at $J= \pm S$ ), $x_{ \pm S}$, can be calculated from (1) to any degree of accuracy by an iteration procedure. Putting $X=x_{0}$ in $f(x, A)$ on the right-hand-side of equation (1) and integrating twice we get the

1. K. Symon, KRS (MURA) $-1,2$, and 4 . 
zeroth order approximation

$$
x(d)=x_{0}+x_{0}^{\prime} d+\int_{0}^{d} f\left(x_{0} \cdot d\right) d d^{2}
$$

Substituting this approximate expression of $x$ into $f(x, d)$, expanding $f(x, A)$ about $x_{0}$ by a Taylor expansion to the first degree term, and integrating the resulting equation twice we get the first order approximate solution

$$
\begin{aligned}
& x(A)=x_{1}+\int_{0}^{\infty} f\left(x_{0}, d\right) d D^{2}+\int_{0}^{\infty} f_{x}\left(x_{0} d\right) d \int_{0}^{d} f(x, d) d d^{2} \\
& \left.+x_{0}+\int_{0}^{d} \int_{0}^{d} f(x, d) d d^{2}\right]
\end{aligned}
$$

where the subscript $X$ means partial differentiation with respect to $X$. The next it eration gives, to the second order approximation,

$$
\begin{aligned}
& x(A)=x_{0}+\int_{0}^{\infty} f\left(x_{0}, d\right) d d^{2}+\int_{0}^{A} f_{x}^{A}\left(x_{0}, A\right) d i \int_{0}^{1} f\left(x_{0}, d\right) d d^{2} \\
& +\int_{0}^{1} f_{x}\left(x_{0}, 0\right) d \int_{0}^{2} f_{x}\left(x_{0}, \Delta\right) d \int_{0}^{2} \int_{0}^{1} f\left(x_{0}, d\right) d d^{2}
\end{aligned}
$$

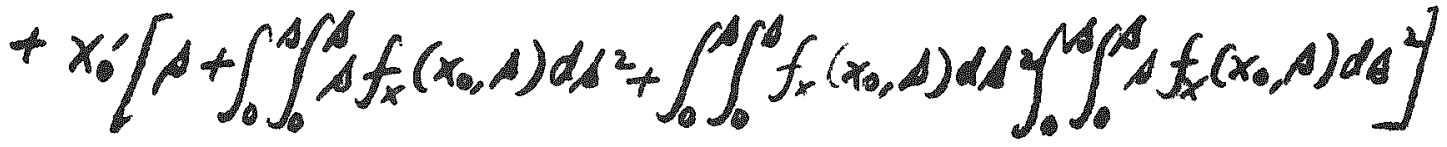

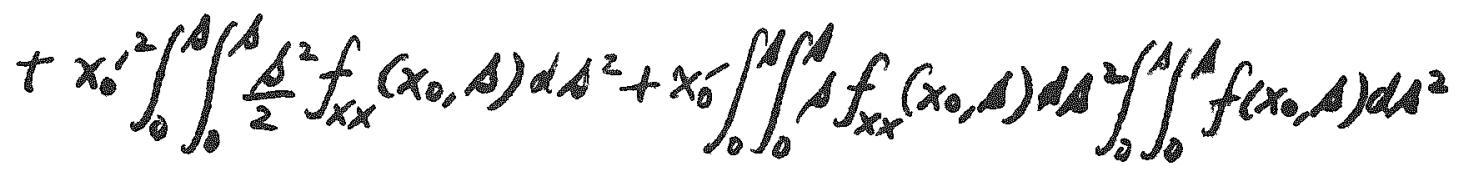

$$
\begin{aligned}
& \left.+\int_{0}^{1} \frac{1}{2} f_{x x}(x, 0) \int_{0}^{1} f\left(x_{0}, d\right) d\right]^{2} d d^{2}
\end{aligned}
$$

This iteration procedure can be carried to any required order of approximation. The resulting series has the same convergence properties as a Taylor series. The smoother $f(x, d)$ as a function of $X$ and the smaller $S\left(\right.$ i.e., the smaller the differences $f\left(x_{S}, 0\right)-f\left(x_{0}, 0\right)$ and $f\left(x_{0}, 0\right)-f\left(x_{g}, 0\right)$, the faster is the convergence of this iteration procedure.

The case of principal interest and to which we shall henceforth restrict ourselves, is when equation (1) has the linear Hill form 
4

$$
x^{\prime \prime}+g(A) x=0
$$

where $g(\&)$ is periodic with period $S$. In this case $f(x, A)=$ $-g(A) \times$ and to the second order approximation, we have

$$
\begin{aligned}
& x_{ \pm S}=x_{0} \Gamma 1-\int_{0}^{ \pm S} \int_{0}^{t} g(A) d s^{2}+\int_{0}^{t S} \int_{0}^{A} g(s) d s^{2} \int_{0}^{s} \int_{0}^{A} g(t) d s^{2} \\
& \left.-\int_{0}^{ \pm S} \int_{0}^{S} g(A) d A^{2} \int_{0}^{s} \int_{0}^{A} g(A) d A^{2} \int_{0}^{A} \int_{0}^{A} g(A) d A^{2}\right] \\
& +x_{0}^{\prime}\left[ \pm S-\int_{0}^{ \pm S} \int_{0} s g(A) d A^{2}+\int_{0}^{t S} \int_{0}^{S} g(A) d A^{2} \int_{0}^{s} \int_{0}^{s} s g(\Delta) d s^{2}\right]
\end{aligned}
$$

The corresponding expression to any order of approximation is selfevident.

As mentioned above, in or der that the "smoothed" curve should coincide with the "exact" curve when the latter is itself smooth, the three point local fitting must be of the same type as the "exact" curve when $g(\delta)$ is a constant; namely, in our present case, a sine curve ${ }^{2}$. Therefore we shall employ a local sine fit and write locally.

$$
Z(s)=A \sin (\Omega s+\alpha)
$$

Thus, we have

$$
\bar{X}(0) \equiv x_{0}=A \sin \alpha, \bar{X}( \pm \beta) \equiv x_{ \pm S}=A \sin ( \pm \Omega, S+\alpha)
$$

2. Here we see that the appropriate local fitting cannot be determined before the nature of the exact solution of $(1)$ is known. To employ arbitrarily a local parabolic fit as was done by Symon, will necessarily lead to erroneous results. In this case the error manifests itself by resulting in the "smoothed" differential equation an $S$ dependent equation (12)). 
5

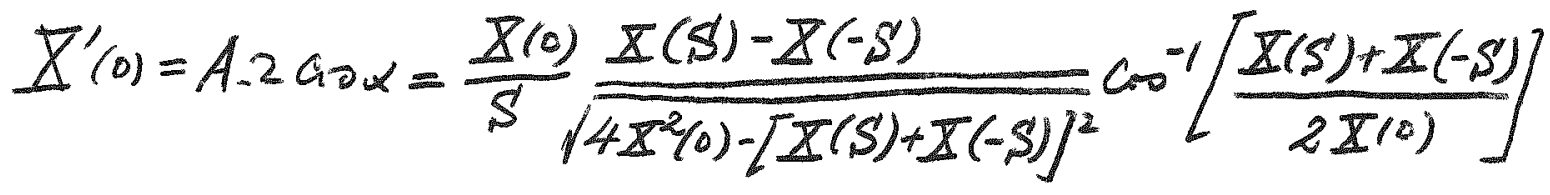

$$
\begin{aligned}
& =\frac{x_{0}}{S} \frac{x_{S}-x_{-S}}{\sqrt{4 x_{0}^{2}-\left[x_{S}+x_{-S}\right]^{2}}} \cos ^{-1}\left(\frac{x_{S}+x_{-S}}{2 x_{0}}\right) \\
& \left.\left.\bar{X}^{\prime \prime}(0)=-A \Omega^{2} \sin x=-\frac{\bar{X}(1)}{S^{2}}\right] \cos ^{-1} \frac{\bar{X}\left(S^{\prime}\right)+\bar{X}(-S)}{2 X(0)}\right]^{2} \\
& =-\frac{x_{2}}{S^{2}}\left[\cos ^{-1} \frac{x_{S}+x_{S S}}{2 x_{0}}\right]^{2}
\end{aligned}
$$

(10)

Substituting $(6)$ and $x_{c}=\bar{X}(0) \quad$ into (10) and striking out the locality-designating symbol $(0)$ in $\bar{X}(0)$ and $\bar{X}^{\prime \prime}(0)$ we get

$$
\begin{aligned}
& X^{\prime \prime}=-\frac{X}{S^{2}}\left\{\omega _ { 0 } { } ^ { - 1 } \left[1-\left(\int_{0}^{S^{2}} \int_{0}^{s} g d A^{2}-\int_{0}^{S \pm} \int_{0}^{A} g d s^{2} \int_{0}^{s} \int_{0}^{s} g d s^{2}\right.\right.\right. \\
& \left.+\int_{c}^{S} \int_{0}^{S \pm} g d s^{2} \int_{0}^{A} \int_{0}^{S} g d x^{2} \int_{0}^{T} \int_{0}^{S} g d s^{2}\right) \\
& \left.\left.-\frac{x_{e}^{\prime}}{X}\left(\int_{0}^{S \pm} \int_{0}^{s} \Delta g d s^{2}-\int_{0}^{s \pm} \int_{0}^{s} g d s^{2} \int_{0}^{s} \int_{0}^{s} s g d s^{2}\right)\right]\right\}^{2}
\end{aligned}
$$

(11)

where $\int_{0}^{S x}$ denotes $\frac{1}{2}\left(\int_{e}^{s}+\int_{0}^{-\infty}\right)$ 
Equation (11) still contains the "un-smoothed" variable $x_{0}^{\prime}$ which should properlybe expressed in terms of $\mathbb{Z}^{\prime}(0)$ and $\bar{X}(0)$ through the equation obtained by substituting (6) in (9). This lengthy procedure is fortunately unnecessary because of the following consideration. In the absence of the $x^{\prime}$ term in the "exact" equation (5), Liouville's theorem states that the $x x^{\prime}$ phase plane area is an invariant of motion. The nature of the correspondence between the set of "smoothed" curves and the set of "exact" curves shows that the $\bar{X} \bar{X}^{\prime}$ phase plane area is also an invariant of motion. This, in turn, indicates that the $\bar{\nabla}^{\prime}$ term will not appear in the properly constructed "smoothed" equation. Therefore, the coefficient of the $x_{0}^{\prime}$ term in (11) should vanish identically to any order of approximation. ${ }^{3}$ This is easily shown to be true by expanding the arc cosine function and evaluating the integrals with the procedure sketched below. Furthermore, as stated earlier, the nature of the "smoothed" equation requires the coefficient of $X$ to be independent of $S$ to any order of approximation. This can also be shown by direct calculation. The "smoothed" equation to any order of approximation is now

$$
X^{\prime \prime}+\omega^{2} \bar{X}=0
$$

where

$$
\omega=\frac{1}{S} \cos ^{-1}(1-\delta)=\text { independent of } S
$$

and

$$
\begin{aligned}
& \delta=\int_{0}^{S^{\prime}} \int_{0}^{A} g d A^{2}-\int_{0}^{S^{ \pm}} \int_{0}^{A} g d d^{2} \int_{0}^{A} \int_{0}^{A} g d A^{2} \\
& +\int_{0}^{S \pm} \int_{0}^{s} g d s^{2} \int_{0}^{s} \int_{0}^{s} g d s^{2} \int_{0}^{s} \int_{0}^{s} g d s^{2}-\cdots
\end{aligned}
$$

The bothersome arc cosine function can be eliminated by noting that since $\omega$ is independent of $\$$ we must have

$$
1-\delta=\cos \omega=1-\frac{\omega^{2} S^{2}}{2 !}+\frac{\omega^{4} S^{4}}{4 !}-\ldots
$$

or

$$
\lim _{s \rightarrow 0} \frac{2 \delta}{S^{2}}=\lim _{s \rightarrow 0} \omega^{2}\left(1-\frac{2 \omega^{2} s^{2}}{4 !}+\frac{2 \omega^{4} s^{4}}{6 !}-\ldots\right)=\omega^{2}
$$

3. The argument Symon gave of making the coefficient of $x_{0}^{\prime}$ vanish to the first order approximation by properly choosing the reference point within a given period, is unnecessary and incorrect. 
7

i.e.,

$$
\omega^{2}=\lim _{s \rightarrow 0} \frac{2 d}{s^{2}}
$$

To further simplify the occurring integrals we introduce the following notation. For any periodic function $F(\&)$ with period $S$ we write

$$
F(A) \equiv \bar{F}+F_{0}(A)
$$

and

$$
F_{i+1}(A) \equiv \int F_{j}(A) d A+C_{j+1} \quad(j=0,1,2,3, \cdots)
$$

where

$$
\bar{F} \equiv \frac{1}{S} \int_{0}^{S} F(S) d S=\text { average of } F(S)
$$

and the constants of integration $C_{j+1}$ are chosen in such a way that $\bar{F}_{i+1}=0$. The functions $F_{j+1}(A)$ so defined are therefore all periodic with period $S$. In addition, we can take advantage of the relations

$$
\begin{aligned}
\overline{g g_{2}} & =\frac{1}{S} \int_{0}^{S} g g_{2} d s=\frac{1}{S}\left[\bar{g} \int_{0}^{S} g_{2} d s+\int_{0}^{S} \frac{d g_{1}}{d s} g_{2} d s\right] \\
& =\frac{1}{S}\left[\left.g_{1} g_{2}\right|_{0} ^{S}-\int_{0}^{S} g_{1} \frac{d g_{2}}{d s} d s\right]=-\frac{1}{S} \int_{0}^{S} g_{1}^{2} d s=-\overline{g_{1}^{2}} \\
\overline{g g_{3}} & =\bar{g} \overline{g_{3}}-\overline{g_{1} g_{2}}=-\overline{g_{1} g_{2}}=\overline{g_{2} g_{1}}=0
\end{aligned}
$$

etc.

$\because 5=7$ 
8

Two examples are worked out in detail below.

$$
\begin{aligned}
& \int_{0}^{S \pm} \int_{0}^{s} g d s^{2}=\bar{g} \int_{0}^{S t} s d s+\int_{0}^{S t} \int_{0}^{s} \frac{d g}{d s} d s^{2} \\
& =\bar{g} \frac{\beta^{2}}{2 !}+\int_{0}^{S^{\prime} t} g_{1} d s-g_{1}(0) \int_{0}^{\beta \pm} d A=\bar{g} \frac{\beta^{2}}{2 !} \\
& \int_{0}^{\beta^{s}} \int_{0}^{s} g d s^{2} \int_{0}^{s} \int_{0}^{s} g d s^{2}=\bar{g} \int_{0}^{s^{s} \pm} \int_{0}^{s} \frac{s^{2}}{2 !} g d s^{2}+\int_{0}^{s} \int_{0}^{s} g g_{2} d x^{2} \\
& -g_{2}(0) \int_{0}^{s \pm} \int_{0}^{s} g d s^{2}-g_{1}(0) \int_{0}^{\beta \Delta} \int_{0}^{s} s g d \beta^{2} \\
& =\bar{g}\left[\bar{g} \int_{0}^{S t} \frac{\Delta^{3}}{3 !} d s+\int_{0}^{S t} \frac{\Delta^{2}}{2 !} g_{1} d s-\int_{0}^{S t} s g_{2} d s+\int_{0}^{S t} g_{3} d s\right. \\
& \left.-g_{3}(0) \int_{0}^{S S t} d \cdot\right]+\bar{g} g_{2} \frac{s^{2}}{2 !}-\bar{g} g_{2}(0) \frac{s^{2}}{2 !} \\
& -g_{1}(0)\left[\bar{g} \int_{0}^{\beta A^{2}} \frac{d^{2}}{2 !} d s+\int_{0}^{S t} \Delta g_{1} d s-\int_{0}^{\beta \pm} g_{2} d s+g_{2}(0) \int_{0}^{S t} d s\right] \\
& =\bar{g}^{2} \frac{S^{4}}{4 !}+\bar{g} g_{2}(0) \frac{S^{12}}{2 !}+\bar{g} g_{2} \frac{S^{2}}{2 !}-\bar{g} g_{2}(0) \frac{S^{2}}{2 !} \\
& =\bar{g}^{2} \frac{S^{4}}{4 !}+\bar{g} \bar{g}_{2} \frac{S^{2}}{2 !}=\bar{g}^{2} \frac{S^{4}}{4 !}-\overline{g_{1}^{2}} \frac{S^{2}}{2 !}
\end{aligned}
$$

The final expression for $\omega^{2}$ to the third order approximation is

$$
\begin{aligned}
& \omega^{2}=\bar{g}+\overline{g_{1}^{2}}+\left(3 \bar{g} \overline{g_{2}^{2}}+\overline{g g_{2}^{2}}\right) \\
& +\left(5 \bar{g}^{-2} \overline{g_{3}^{2}}+4 \bar{g} \overline{g g_{3}^{2}}+6 \bar{g}\left(\overline{\left.g g_{2}\right)_{1} g_{3}}+3 \overline{g_{1}^{2}} \overline{g_{2}^{2}}+\overline{\left(g g_{2}\right)_{1}^{2}}\right)\right. \\
& +\cdots
\end{aligned}
$$

(14) 
From the above prescription it is clear that $\omega$ given by (14) is nothing but the(angular) frequency of the dominating Fourier component of the solution of equation (5). The replacement of the "exact" curve by the "smoothed" curve offers a natural and exact definition of the frequency (or wave length) of the gross oscillation for all values of $S$ and $\omega$. The wave length $\frac{2 \pi}{\omega}$ so derived coincides with that obtained from the definition

wave length $\equiv \lim _{\Delta \rightarrow \infty}$

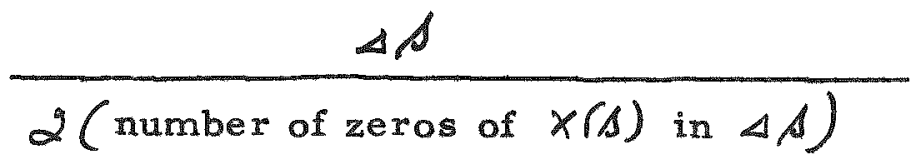

irrespective of the relative magnitudes of $S$ and $\frac{2 \pi}{\omega}$. However, the rate of convergence of the iteration procedure used to derive $x_{ \pm S}$ as functions of $x_{0}$ and $X_{0}^{\prime}$ depends on the ratio $\frac{2 \pi S}{\omega}$, being faster for smaller. $\frac{2 \pi S}{\omega}$.

Example and Check

In the special case of an alternating gradient synchrotron when the half sector lengths are equal and $n_{1}=-n_{2}=n(n$ being the usual magnetic field index $\frac{r}{8} \frac{\partial b}{\partial r}$ ) the frequency of the $z$-oscillation given by the exact matrix calculation is .

or

$$
\cos \rho_{j} \omega_{z}=\cos \sqrt{x} S \cosh \sqrt{n} S
$$

$$
\omega_{z}^{2}=\frac{1}{4 s^{2}}\left[\cos ^{-1}(\cos \sqrt{n} s \operatorname{coh} \sqrt{n} s)\right]^{2}
$$

where the sector length (period) is, for convenience, taken to be $2 S$ instead of $S$. Expanding the trigonometric functions in powers of $n S^{2}$ we get

$$
\omega_{z}^{2}=\frac{n^{2} s^{2}}{12}+\frac{2}{945} n^{4} s^{6}+\cdots \cdots
$$

Now to calculate $\omega_{z}$ by (14), the function $q(\mathcal{A})$ is, in this case

4. e.g. E. D. Courant and H. S. Snyder, Brookhaven National Laboratory Report EDC/HSS-1 
10

$$
g(s)=\left\{\begin{array}{cc}
x & 0<\beta<S \\
-n & -S<\beta<0
\end{array}\right.
$$

Since here, $\bar{g}=0$ the "smooth approximation" for mull (14) becomes

$$
\omega_{z}^{2}=\overline{g_{1}^{2}}+\overline{g g^{2}}+\left[\overline{3} \overline{g_{1}^{2}} \overline{g_{2}^{2}}+\overline{\left(g g_{2}\right)_{1}^{2}}\right]
$$

The various terms on the right-hand side are calculated below.

$$
\begin{aligned}
& g_{1}(A)=\left\{\begin{array}{l}
n A-\frac{n S}{2} \\
-n A-\frac{n S}{2}
\end{array} \quad g_{1}^{2}=\left\{\begin{array}{l}
n^{2} A^{2}-n^{2} S A+\frac{n^{2} S^{2}}{4} \\
n^{2} A^{2}+n^{2} S A+\frac{n^{2} S^{2}}{4}
\end{array}\right.\right. \\
& q_{2}(A)=\left\{\begin{array}{l}
\frac{n}{2} A^{2}-\frac{n S}{2} A \\
-\frac{n}{2} A^{2}-\frac{n S}{2} A
\end{array} \quad g_{2}^{2}=\left\{\begin{array}{l}
\frac{x^{2}}{4} A^{4}-\frac{x^{2} S^{4}}{2} A^{3}+\frac{x^{2} S^{2}}{4} A^{2} \\
\frac{x^{2}}{4} A^{4}+\frac{x^{2} S}{2} A^{3}+\frac{x^{2} S^{2}}{4} A^{2}
\end{array}\right.\right. \\
& g g_{2}^{2}=\left\{\begin{array}{l}
\frac{n^{3}}{4} s^{4}-\frac{x^{3} S}{2} s^{3}+\frac{n^{3} S^{2}}{4} s^{2} \\
-\frac{n^{3}}{4} s^{4}-\frac{n^{3} S}{2} s^{3}-\frac{n^{3} S^{2}}{4} s^{2}
\end{array}\right. \\
& g_{2}=\left\{\begin{array}{l}
\frac{x^{2}}{2} A^{2}-\frac{x^{2} S}{2} A \\
\frac{x^{2}}{2} A^{2}+\frac{x^{2} S}{2} A
\end{array}\right. \\
& g g_{2}-\overline{g g_{2}}=\left\{\begin{array}{l}
\frac{x^{2}}{2} A^{2}-\frac{x^{2} S}{2} A+\frac{x^{2} S^{2}}{12} \\
\frac{x^{2}}{2} A^{2}+\frac{x^{2} S}{2} A+\frac{x^{2} S^{2}}{12}
\end{array}\right.
\end{aligned}
$$


11

$$
\begin{aligned}
& \left(g q_{2}\right)_{1}=\left\{\begin{array}{l}
\frac{x^{2}}{6} A^{3}-\frac{x^{2} S}{4} s^{2}+\frac{x^{2} S^{2}}{12} s \\
\frac{x^{2}}{6} A^{3}+\frac{x^{2} s}{4} s^{2}+\frac{x^{2} S^{2}}{12} s
\end{array}\right. \\
& \left(g g_{2}\right)_{1}^{2}=\left\{\begin{array}{l}
\frac{x^{4}}{36} s^{6}-\frac{x^{4} S}{12} s^{5}+\frac{13 x^{4} S^{2}}{144} s^{4}-\frac{x^{4} S^{3}}{24} s^{3}+\frac{x^{4} S^{4}}{744} A^{2} \\
\frac{x^{4}}{36} s^{6}+\frac{x^{4} S}{12} s^{5}+\frac{13 x^{4} S^{2}}{144} s^{4}+\frac{x^{4} S^{3}}{24} s^{3}+\frac{x^{4} S^{4}}{144} s^{2}
\end{array}\right.
\end{aligned}
$$

$$
\begin{aligned}
& \overline{g_{1}^{2}}=x^{2} S^{2}\left(\frac{1}{3}-\frac{1}{2}+\frac{1}{4}\right)=\frac{x^{2} S^{2}}{12} \quad \overline{g g^{2}}=0 \\
& \overline{g_{2}}=x^{2} s^{4}\left(\frac{1}{20}-\frac{1}{8}+\frac{1}{12}\right)=\frac{x^{2} s^{4}}{120} \quad 3 \overline{g_{1}^{2}} \overline{g_{2}^{2}}=\frac{x^{4} s^{6}}{480} \\
& \overline{\left(g g_{2}\right)_{1}^{2}}=x^{4} S^{6}\left(\frac{1}{252}-\frac{1}{72}+\frac{13}{720}-\frac{1}{96}+\frac{1}{432}\right)=\frac{x^{4} s^{6}}{30240}
\end{aligned}
$$

Therefore

$$
\begin{aligned}
\omega_{z}^{2} & =\overline{g_{1}^{2}}+\overline{g g_{2}^{2}}+\left[3 \overline{g_{1}^{2}} \overline{g_{2}^{2}}+\overline{\left(g g_{2}\right)_{1}^{2}}\right]+\ldots . \\
& =\frac{x^{2} s^{2}}{12}+\frac{2}{945} x^{4} s^{6}+\ldots .
\end{aligned}
$$

which agrees with the exact result (15). 